\title{
THE DOUBLE LOGARITHM AND MANIN'S COMPLEX FOR MODULAR CURVES
}

\section{A. B. Goncharov}

To Sasha Beilinson for his 40-th birthday

\section{Introduction}

Multiple polylogarithms are defined via power series expansion:

$$
L i_{n_{1}, \ldots, n_{m}}\left(x_{1}, \ldots, x_{m}\right)=\sum_{0<k_{1}<k_{2}<\ldots<k_{m}} \frac{x_{1}^{k_{1}} x_{2}^{k_{2}} \ldots x_{m}^{k_{m}}}{k_{1}^{n_{1}} k_{2}^{n_{2}} \ldots k_{m}^{n_{m}}} .
$$

Here $w:=n_{1}+\ldots+n_{m}$ is called the weight and $m$ the depth of the multiple polylogarithm. These power series obviously generalize both Euler's classical polylogarithms $L i_{n}(x)(\mathrm{m}=1)$, and Euler's sums [E], often called multiple $\zeta$ numbers $\left(x_{1}=\ldots=x_{m}=1\right)$ and studied in [Dr], [Z1-3], [G3-4], ...:

$$
\zeta\left(n_{1}, \ldots, n_{m}\right):=\sum_{0<k_{1}<k_{2}<\ldots<k_{m}} \frac{1}{k_{1}^{n_{1}} k_{2}^{n_{2}} \ldots k_{l}^{n_{m}}} \quad n_{m}>1 .
$$

Multiple polylogarithms are periods of mixed Tate motives (s.12 of [G2], [G3]).

Let $\Gamma_{1}(N ; m) \subset G L_{m}(\mathbb{Z})$ be the subgroup of the matrices whose bottom row is congruent to $(0, \ldots, 0,1)$ modulo $N$ and $V_{m}$ the standard $m$-dimensional representation of $G L_{m}(\mathbb{Z})$. In this note we begin to study a mysterious connection between the multiple polylogarithms of depth $m$ at $N$-th roots of unity and cohomology of $\Gamma_{1}(N ; m)$ with coefficients in $S^{w-m}\left(V_{m}\right)$ where $m>1$. We will work out in details the simplest case: $m=2, n_{1}=n_{2}=1, p$ is prime. In s. 4 we touch the general situation and will return to it in [G1], see also [G4].

So we investigate the double logarithm function

$$
L i_{1,1}(x, y)=\sum_{0<k_{1}<k_{2}} \frac{x^{k_{1}} y^{k_{2}}}{k_{1} k_{2}}
$$

The series are convergent for $|x|,|y| \leq 1,(x, y) \neq(1,1)$. They admit analytic continuation to a multivalued analytic function.

We show that the double logarithm is a period of a mixed Tate motive $\tilde{L} i_{1,1}(x, y)$ equipped with an additional data: 2 -framing. Let $p$ be a prime. If

Received May 30, 1997.

Research supported by NSF grant DMS-9500010. 
$x$ and $y$ are $p$-th roots of unity the corresponding mixed Tate motive is defined over the scheme $S_{p}:=\operatorname{Spec} \mathbb{Z}[x] /\left(x^{p}-1\right)$.

The 2-framed mixed motives form a $\mathbb{Q}$-vector space. Let $\mathcal{L}(p)_{2}$ be the space generated by all 2 -framed mixed Tate motives over the scheme $S_{p}$, and $\mathcal{C}(p)_{2} \otimes \mathbb{Q}$ the subspace genertated by the motives $\tilde{L} i_{1,1}(x, y), x^{p}=y^{p}=1$.

Let $C_{p}$ be the group of cyclotomic units in $\mathbb{Z}\left[\zeta_{p}\right]$. We will construct a complex

$$
\delta: \mathcal{C}(p)_{2} \otimes \mathbb{Q} \rightarrow \Lambda^{2} C_{p} \otimes \mathbb{Q}
$$

which encodes the essential information about the double logarithm at $p$-th roots of unity. One can show that $\operatorname{Ker} \delta=K_{3}\left(\mathbb{Z}\left[\zeta_{p}\right]\right) \otimes \mathbb{Q}$.

Consider the classical triangulation of the hyperbolic plane by the images of the geodesic triangle with vertices at $0,1, \infty$ under the action of $S L_{2}(\mathbb{Z})$. Projecting it down we get canonical triangulation of a modular curve $X$. By definition Manin's complex $C_{\bullet}(X)$ is the chain complex of this triangulation.

We will construct a natural correspondence between the numbers $L i_{1,1}\left(\zeta_{p}^{a}, \zeta_{p}^{b}\right)$ and the triangles on the modular curve $X_{1}(p)$. More precisely, let $C_{\bullet}\left(X_{1}(p)\right)_{+}$ be the coinvariants of the complex conjugation acting on the complex points of $X_{1}(p)$. We will construct a canonical isomorphism between (truncated) Manin's complex $\tau_{[2,1]} C_{\bullet}\left(X_{1}(p)\right)_{+}$and the quotient of complex (4) along Ker $\delta$. This implies that $\mathcal{L}(p)_{2} / \mathcal{C}(p)_{2} \otimes \mathbb{Q}$ is canonically isomorphic to $H_{1}\left(X_{1}(p), \mathbb{Q}\right)_{+}$.

One may compare this with the classical theory of cyclotomic units, where $\{$ units $\} /\{$ cyclotomic units $\}$ is a finite group of order $h_{p}^{+}$(the plus part of the class number of of the cyclotomic field). Notice that $L_{1}(x)=-\log (1-x)$, so restricting $x$ to the $p$-th roots of unity we get $-\log \left(1-\zeta_{p}^{\alpha}\right)$. Thus we consider $\mathcal{L}(p)_{2}$ as the "weight 2 " analog of the group of units $\mathbb{Z}\left[\zeta_{p}\right]^{*}$, and $\mathcal{C}(p)_{2}$ as an analog of the subgroup of the cyclotomic units.

We suggest that the "higher cyclotomy theory" should study the multiple polylogarithm motives at roots of unity.

\section{The double logarithm}

1. Properties of the double logarithm function. (See [G1]-[G4] for general properties of multiple polylogarithms). Consider the following iterated integral:

$$
I_{1,1}\left(a_{1}, a_{2}\right):=\int_{0}^{1} \frac{d t}{t-a_{1}} \circ \frac{d t}{t-a_{2}}:=\iint_{0<t_{1}<t_{2}<1} \frac{d t_{1}}{t_{1}-a_{1}} \wedge \frac{d t_{2}}{t_{2}-a_{2}} .
$$

Key Lemma. The double logarithm can be written as an iterated integral:

$$
L i_{1,1}(x, y)=\int_{0<t_{1}<t_{2}<1} \frac{d t_{1}}{t_{1}-(x y)^{-1}} \frac{d t_{2}}{t_{2}-y^{-1}}=I_{1,1}\left((x y)^{-1}, y^{-1}\right) .
$$


Proof. Using $\frac{d t}{t-a}=\sum_{k=1}^{\infty} \frac{t^{k-1}}{a^{k}}$ we get

$$
\begin{gathered}
I_{1,1}\left(a_{1}, a_{2}\right)=\int_{0}^{1}\left(\int_{0}^{t_{2}}\left(\sum_{k_{1}=1}^{\infty} \frac{t_{1}^{k_{1}-1}}{a_{1}^{k_{1}-1}}\right) d t_{1}\right) \cdot\left(\sum_{k_{2}=1}^{\infty} \frac{t_{2}^{k_{2}-1}}{a_{2}^{k_{2}-1}}\right) d t_{2}= \\
\sum_{k_{1}, k_{2}=1}^{\infty} \frac{1}{k_{1}} \int_{0}^{1} \frac{t_{2}^{k_{1}+k_{2}-1}}{a_{1}^{k_{1}} a_{2}^{k_{2}}} d t_{2}=\sum_{k_{1}, k_{2}=1}^{\infty} \frac{\left(a_{2} / a_{1}\right)^{k_{1}} \cdot\left(1 / a_{2}\right)^{k_{1}+k_{2}}}{k_{1}\left(k_{1}+k_{2}\right)} .
\end{gathered}
$$

This lemma provides an analytic continuation of the double logarithm.

Symmetry relations. The double logarithm enjoys the following properties:

$$
\begin{gathered}
\log (1-x) \log (1-y)=L i_{1,1}(x, y)+L i_{1,1}(y, x)+L i_{2}(x y) \\
\log \left(1-x^{-1}\right) \log \left(1-y^{-1}\right)=I_{1,1}(x, y)+I_{1,1}(y, x)
\end{gathered}
$$

(Notice that $I_{1,1}(x, y)+I_{1,1}(y, x)=L i_{1,1}\left(\frac{y}{x}, \frac{1}{y}\right)+L i_{1,1}\left(\frac{x}{y}, \frac{1}{x}\right)$ ).

Indeed, multiplying the power series for $\log (1-x)$ and $\log (1-y)$ we get

$$
\left(\sum_{0<k_{1}<k_{2}}+\sum_{0<k_{1}=k_{2}}+\sum_{0<k_{2}<k_{1}}\right) \frac{x^{k_{1}} y^{k_{2}}}{k_{1} k_{2}}
$$

which is just the right hand side of the first identity.

The second follows from the product formula for iterated integrals:

$$
\int_{0}^{1} \frac{d t}{t-x} \cdot \int_{0}^{1} \frac{d t}{t-y}=\int_{0}^{1} \frac{d t}{t-x} \circ \frac{d t}{t-y}+\int_{0}^{1} \frac{d t}{t-y} \circ \frac{d t}{t-x} .
$$

The distribution relations. For any $n \mid N$ and $|x|,|y| \leq 1$ one has

$$
L i_{1,1}\left(x_{1}, x_{2}\right)=\sum_{y_{i}^{n}=x_{i}} L i_{1,1}\left(y_{1}, y_{2}\right), \quad L i_{2}(x)=n \cdot \sum_{y^{n}=x} L i_{2}(y) .
$$

This is easy to show using the power series expansion.

The differential equation. One has

$$
d L i_{1,1}(x, y)=\log (1-x y) d \log \frac{(1-y) x}{(1-x)}+\log (1-y) d \log (1-x) .
$$

Indeed,

$$
\begin{gathered}
d L i_{1,1}(x, y)=\sum_{0<k_{1}<k_{2}}\left(\frac{x^{k_{1}}}{k_{1}} y^{k_{2}-1} d y+x^{k_{1}-1} \frac{y^{k_{2}}}{k_{2}} d x\right)= \\
\sum_{0<k_{1}} \frac{x^{k_{1}}}{k_{1}} \frac{y^{k_{1}}}{1-y} d y+\sum_{0<k_{2}} \frac{\left(x^{k_{2}-1}-1\right)}{x-1} \frac{y^{k_{2}}}{k_{2}} d x= \\
\log (1-x y) d \log (1-y)-\log (1-x y) \frac{d x}{x(x-1)}+\log (1-y) d \log (1-x) .
\end{gathered}
$$

Using $d x / x(x-1)=(1 /(x-1)-1 / x) d x$ we get the formula. 
Relation with the dilogaritm. It is easy to check by differentiation that

$$
L i_{1,1}(x, y)=L i_{2}\left(\frac{x y-y}{1-y}\right)-L i_{2}\left(\frac{y}{y-1}\right)-L i_{2}(x y) .
$$

Substituting this to (5) we get the famous five term relation for the dilogarithm:

$$
\begin{gathered}
L i_{2}\left(\frac{x y-y}{1-y}\right)+L i_{2}\left(\frac{x y-x}{1-x}\right)-L i_{2}\left(\frac{y}{y-1}\right)-L i_{2}\left(\frac{x}{x-1}\right)-L i_{2}(x y)= \\
\log (1-x) \cdot \log (1-y) .
\end{gathered}
$$

2. A variation of mixed Hodge structures related to the double logarithm. Let

$$
A_{1,1}(x, y):=\left(\begin{array}{cccc}
1 & 0 & 0 & 0 \\
\log (1-x y) & 2 \pi i & 0 & 0 \\
\log (1-y) & 0 & 2 \pi i & 0 \\
L i_{1,1}(x, y) & 2 \pi i \cdot \log \frac{(1-y) x}{(1-x)} & 2 \pi i \cdot \log x & (2 \pi i)^{2}
\end{array}\right) .
$$

The matrix $A_{1,1}(x, y)$ defines a variation of mixed Hodge structures over $\mathbb{C}^{2} \backslash\{x=$ $0, x=1, y=1, x y=1\}$ as follows.

Let $C_{i}$ be the $i$-th column of the matrix $A_{1,1}(x, y), i=0, \ldots, 3$. For given $(x, y)$ let $H_{1,1}(x, y):=<C_{0}, \ldots, C_{3}>_{\mathbb{Q}}$ be the $\mathbb{Q}$-linear combinations of columnes of the matrix $A_{1,1}(x, y)$. The monodromy properties of the function $L i_{1,1}(x, y)$ and logarithms imply that $\mathbb{Q}$-vector spaces $H_{1,1}(x, y)$ form a local system, called $H_{1,1}$. It has a weight filtration defined as follows:

$$
W_{-2 k} H_{1,1}(x, y):=<C_{k}, \ldots, C_{3}>, \quad W_{-2 k+1}=W_{-2 k} .
$$

Let $e_{0}, \ldots, e_{3}$ be the standard basis in $\mathbb{C}^{4}$ (the space of columnes). Then $H_{1,1}(x, y) \otimes_{\mathbb{Q}} \mathbb{C}=\mathbb{C}^{4}$. We define the Hodge filtration setting $F^{-k} H_{1,1}(x, y)=$ $<e_{0}, \ldots, e_{k}>\mathbb{C}$.

Lemma 2.1. $H_{1,1}$ is a variation of mixed Hodge structures.

Proof. The Griffith transversality condition is just equivalent to the differential equation for the double logarithm function.

3. A 2-framed mixed Tate motive $\tilde{I}_{1,1}\left(a_{1}, a_{2}\right)$. Changing variables $u_{i}:=$ $t_{i}-a_{i}$ we get

$$
I_{1,1}\left(a_{1}, a_{2}\right):=\iint_{\Delta\left(a_{1}, a_{2}\right)} \frac{d u_{1}}{u_{1}} \wedge \frac{d u_{2}}{u_{2}},
$$

where for appropriate real $\left(a_{1}, a_{2}\right)$ one has

$$
\Delta\left(a_{1}, a_{2}\right):=\left\{\left(u_{1}, u_{2}\right) \subset \mathbb{R}^{2} \mid \quad 0 \leq u_{1}+a_{1} \leq u_{2}+a_{2} \leq 1\right\} .
$$

This suggests the following interpretation of the double logarithms as a period of a mixed Tate motive (More general motives were studied in [BMSV]).

Let $\left(u_{0}: u_{1}: u_{2}\right)$ be homogeneous coordinates in $\mathbb{P}^{2}, L_{i}=\left\{u_{i}=0\right\}$ the coordinate lines and $L:=L_{0} \cup L_{1} \cup L_{2}$ the coordinate triangle. 
Set $M:=M_{0} \cup M_{1} \cup M_{2}$ where

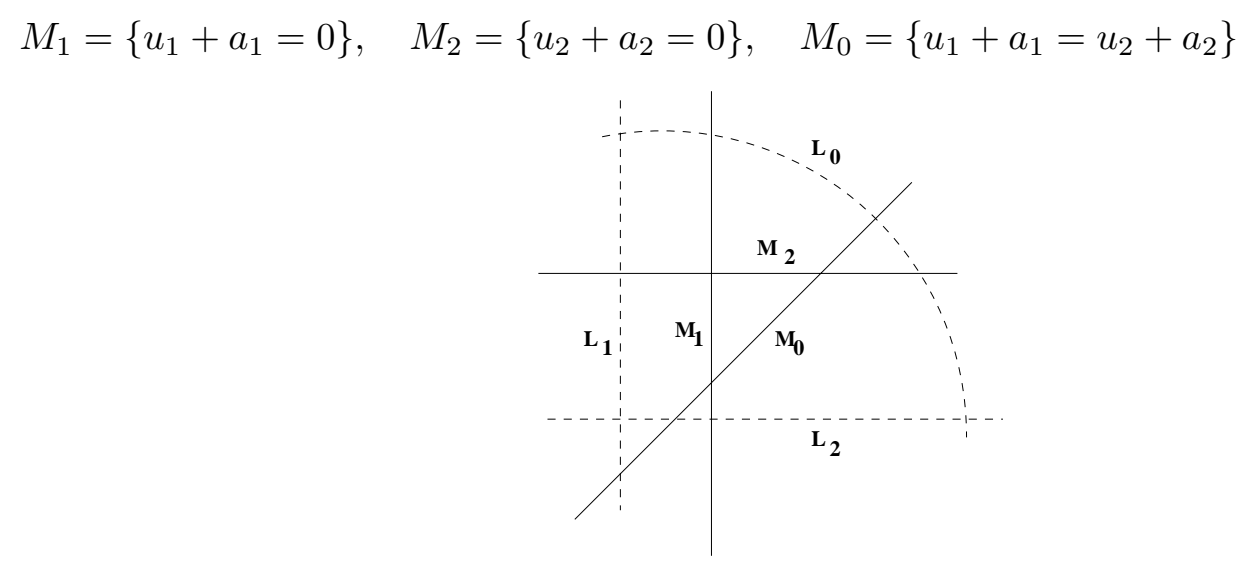

Let $\Delta_{M}$ be an oriented 2-chain in $\mathbb{C P}^{2} \backslash L$, which is the homeomorphic image of the triangle $\left(t_{1}, t_{2}\right) \mid t_{1} \geq 0, t_{2} \geq 0, t_{1}+t_{2} \leq 1$, with sides on lines $M_{i}$. Its vertices coinside with the ones of $M$. Then $\Delta_{M}$ represents a generator of the group $H_{2}\left(\mathbb{C P}^{2}, M ; \mathbb{Z}\right)$. The formula $I_{1,1}\left(a_{1}, a_{2}\right)=\int_{\Delta_{M}} \omega_{L}$ provides an analytic continuation of the double logarithm.

Consider the mixed Hodge structure

$$
H_{L, M}:=H^{2}\left(\mathbb{C P}^{2} \backslash L, M\right) .
$$

The only non zero Hodge numbers are $h^{0,0}, h^{1,1}, h^{2,2}$, and in general $h^{0,0}=$ $1, h^{1,1}=2, h^{2,2}=1$.

The natural map $H_{L, M} \rightarrow H^{2}\left(\mathbb{C P}^{2} \backslash L\right)$ provides an isomorphism $g r_{4}^{W} H_{L, M} \longrightarrow H^{2}\left(\mathbb{C P}^{2} \backslash L\right)=\mathbb{Q}(-2)$, and similarly we get an isomorphism $\mathbb{Q}(0)=H^{2}\left(\mathbb{C P}^{2}, M\right) \longrightarrow W_{0} H_{L, M}$. Thus we get distinguished elements

$$
\omega_{L} \in \operatorname{Hom}_{\mathcal{M}}\left(\mathbb{Q}(-2), g r_{4}^{W} H_{L, M}\right), \quad\left[\Delta_{M}\right] \in\left(\operatorname{Hom}_{\mathcal{M}}\left(\mathbb{Q}(0), W_{0} H_{L, M}\right)\right)^{*}
$$

and $\int_{\Delta_{M}} \omega_{L}$ is a period of the mixed Hodge structure $H_{L, M}$.

4. The Tannakian formalism for mixed Tate categories: a review. (see also $[\mathrm{BMS}],[\mathrm{BGSV}])$. Let $\mathcal{M}$ be an abelian tensor $\mathbb{Q}$-category with an invertible object $\mathbb{Q}(1)$. Set $\mathbb{Q}(n):=\mathbb{Q}(1)^{n}$. We will say that $\mathcal{M}$ is a mixed Tate category if the objects $\mathbb{Q}(n)$ are mutually nonisomorphic, any simple object is isomorphic to one of them and $\operatorname{Ext}_{\mathcal{M}}^{1}(\mathbb{Q}(0), \mathbb{Q}(n))=0$ if $n \leq 0$.

Any object $M$ of a mixed Tate category has a canonical weight filtration $W \bullet M$ such that $g r_{2 k}^{W} M=\oplus \mathbb{Q}(-k), g r_{2 k+1}^{W} M=0$. The functor

$$
\omega: \mathcal{M} \longrightarrow \text { Vect• }, \quad M \longmapsto \oplus_{k} \operatorname{Hom}_{\mathcal{M}}\left(\mathbb{Q}(-k), g r_{2 k}^{W} M\right)
$$

to the category of graded $\mathbb{Q}$-vector spaces is a fiber functor. Let

$$
L(\mathcal{M})_{\bullet}:=\operatorname{Der}(\omega):=\left\{F \in \operatorname{End} \omega \mid F_{X \otimes Y}=F_{X} \otimes i d_{Y}+i d_{Y} \otimes F_{Y}\right\}
$$

be the space of its derivations. It is a graded pro-Lie algebra over $\mathbb{Q}$. 
Let $\tilde{\omega}$ be the fiber functor to the category of finite dimensional $\mathbb{Q}$-vector spaces obtained from $\omega$ by forgetting the grading. Then $A u t^{\otimes} \tilde{\omega}$ is a pro-algebraic group scheme over $\mathbb{Q}$. It is a semidirect product of $\mathbb{G}_{m}$ and a pro-unipotent group scheme $G$. The pro-Lie algebra $L(\mathcal{M})$ is the Lie algebra of the group scheme $G$. The action of $\mathbb{G}_{m}$ provides a grading on $L(\mathcal{M})$.

According to the Tannakian formalism the category $\mathcal{M}$ is canonically equivalent to the category of finite dinensional $\mathbb{Q}$-modules over the group scheme $A u t^{\otimes} \tilde{\omega}$. This category is naturally equivalent to the category of graded finite dinensional modules over the group scheme $G$. Since $G$ is pro-unipotent, the last category is equivalent to the category graded finite dinensional modules over the graded pro-Lie algebra $L(\mathcal{M})$.

Let $\mathcal{U}(\mathcal{M})_{\bullet}:=\operatorname{End}(\omega)$ be the space of all endomorphisms of the fiber functor $\omega$. It is a Hopf algebra which is isomorphic to the universal enveloping algebra of the Lie algebra $L(\mathcal{M})$.

The dual Hopf algebra $\left(\mathcal{U}(\mathcal{M})_{\bullet}\right)^{*}$ can be identified with the Hopf algebra $\mathbb{Q}[G]$ of regular functions on $G$. The action of $\mathbb{G}_{m}$ on $G$ provides a grading on it. Below we give a more concrete way to think about this Hopf algebra.

Let $n \geq 0$. Say that $M$ is an $n$-framed object of $\mathcal{M}$ if it is supplied with a nonzero morphisms $v_{n}: \mathbb{Q}(-n) \longrightarrow g r_{2 n}^{W} M$ and $f_{0}: g r_{0}^{W} M \longrightarrow \mathbb{Q}(0)$.

Consider the finest equivalence relation on the set of all $n$-framed objects for which $M_{1} \sim M_{2}$ if there is a map $M_{1} \rightarrow M_{2}$ respecting the frames. For example any $n$-framed object is equivalent to a one $M$ with $W_{-2} M=0, W_{2 n} M=M$. Let $\mathcal{A}_{n}$ be the set of equivalence classes. It is an abelian group:

$$
\left[M, v_{n}, f_{0}\right]+\left[M^{\prime}, v_{n}^{\prime}, f_{0}^{\prime}\right]=\left[M \oplus M^{\prime},\left(v_{n}, v_{n}^{\prime}\right), f_{0}+f_{0}^{\prime}\right]
$$

$-\left[M, v_{n}, f_{0}\right]:=\left[M,-v_{n}, f_{0}\right]=\left[M, v_{n},-f_{0}\right]$. The neutral element is $\mathbb{Q}(0) \oplus$ $\mathbb{Q}(-n)$ with the obvious frame. The composition $f_{0} \circ v_{0}: \mathbb{Q}(0) \rightarrow \mathbb{Q}(0)$ provides an isomorphism $\mathcal{A}_{0}=\mathbb{Q}$.

The tensor product induces the commutative multiplication $\mu: \mathcal{A}_{k} \otimes \mathcal{A}_{\ell} \rightarrow$ $\mathcal{A}_{k+\ell}$. Let us define the comultiplication

$$
\Delta=\bigoplus_{0 \leq k \leq n} \Delta_{k, n-k}: \mathcal{A}_{n} \rightarrow \bigoplus_{0 \leq k \leq n} \mathcal{A}_{k} \otimes \mathcal{A}_{n-k} .
$$

Choose a basis $p_{1}, \ldots, p_{m}$ of $\operatorname{Hom}_{\mathcal{M}}\left(\mathbb{Q}(-k), g r_{2 k}^{W} M\right)$ and the dual basis $p_{1}^{\prime}, \ldots, p_{m}^{\prime}$ of $\left(\operatorname{Hom}_{\mathcal{M}}\left(g r_{2 k}^{W} M, \mathbb{Q}(-k)\right)\right.$. Then

$$
\Delta_{k, n-k}\left[M, v_{n}, f_{0}\right]:=\sum_{i=1}^{m}\left[M, v_{n}, p_{i}^{\prime}\right](n-k) \otimes\left[M, p_{i}, f_{0}\right] .
$$

In particular $\Delta_{0, n}=i d \otimes 1$ and $\Delta_{n, 0}=1 \otimes i d$. Then $\mathcal{A}(\mathcal{M})_{\bullet}:=\oplus \mathcal{A}(\mathcal{M})_{n}$ is a graded Hopf algebra with the commutative multiplication $\mu$ and the comultiplication $\Delta$.

Theorem 2.2. The Hopf algebra $\mathcal{A}_{\bullet}:=\oplus_{k=0}^{\infty} \mathcal{A}_{k}$ is canonically isomorphic to the dual of the Hopf algebra $\mathcal{U}(\mathcal{M})$. 
A canonical morphism $i: \mathcal{A}_{\bullet} \rightarrow \mathcal{U}(\mathcal{M})_{\bullet}^{*}$ is constructed as follows. Let $F \in$ $\operatorname{End}(\omega)_{n}$ and $\left[M, v_{n}, f_{0}\right] \in \mathcal{A}_{n}$. Then $\left\langle i\left(\left[M, v_{n}, f_{0}\right]\right), F>:=<f_{0}, F\left(v_{n}\right)>\right.$.

Set $\Delta^{\prime}(X):=\Delta(X)-(X \otimes 1+1 \otimes X) . \Delta^{\prime}$ provides the quotient $\mathcal{L}(\mathcal{M}) \bullet:=$ $\mathcal{A}(\mathcal{M})_{\bullet} /\left(\mathcal{A}(\mathcal{M})_{>0}\right)^{2}$ with the structure of a Lie coalgebra with cobracket $\delta$.

An example. A $\mathbb{Q}$-Hodge-Tate structure is a mixed $\mathbb{Q}$-Hodge structure with $h^{p, q}=0$ if $p \neq q$. Let $\mathcal{H} \mathcal{T}_{\mathbb{Q}}$ be the category of $\mathbb{Q}$-Hodge-Tate structures. Set $\mathcal{H}_{\bullet}:=\mathcal{A}_{\bullet}\left(\mathcal{H} \mathcal{T}_{\mathbb{Q}}\right)$. Then

$$
\mathcal{H}_{1}=\operatorname{Ext}_{\mathbb{Q}-M H S}^{1}(\mathbb{Q}(0), \mathbb{Q}(1))=\frac{\mathbb{C}}{2 \pi i \mathbb{Q}}=\mathbb{C}_{\mathbb{Q}}^{*} .
$$

Under the isomorphism the extension provided by the mixed Hodge structure given by the columns of the matrix $\left(\begin{array}{cc}1 & 0 \\ \log (z) & 2 \pi i\end{array}\right)$ corresponds to $z \in \mathbb{C}^{*}$.

One has

$$
\Delta^{\prime}: \mathcal{H}_{2} \longrightarrow \mathcal{H}_{1} \otimes \mathcal{H}_{1}=\mathbb{C}^{*} \otimes_{\mathbb{Q}} \mathbb{C}^{*}, \quad \delta: \mathcal{H}_{2} /\left(\mathcal{H}_{1}\right)^{2} \longrightarrow \Lambda_{\mathbb{Q}}^{2} \mathbb{C}^{*}
$$

Below a tilda over $L i$ always means that we are dealing with the framed Hodge-Tate structure related to a multiple polylogarithm. See for the general construction s. 12 of [G2] or [G3].

\section{Proposition 2.3.}

$$
\Delta^{\prime} \tilde{L} i_{1,1}(x, y)=(1-x y) \otimes \frac{(1-y) x}{(1-x)}+(1-y) \otimes(1-x) \in \mathbb{C}^{*} \otimes_{\mathbb{Q}} \mathbb{C}^{*} .
$$

Proof. Follows from the description of the mixed Hodge structure given in $§ 2.2$.

\section{The double logarithm at $N$-th roots of unity and Manin's complex for $X_{1}(N)$}

1. Symmetry relations. In this section we study the 2-framed mixed Hodge structures related to the double logarithm at roots of unity.

Theorem 3.1. Let $a, b$ be $N$-th roots of unity. Then modulo $N$-torsion one has the following relations between the motivic double logarithms:

The symmetry relations:

a) In the depth one:

$$
\tilde{L} i_{1}\left(a^{-1}\right)=\tilde{L} i_{1}(a), \quad \tilde{L} i_{2}\left(a^{-1}\right)+\tilde{L} i_{2}(a)=0 .
$$

b) In the depth two:

$$
\begin{gathered}
\tilde{L} i_{1,1}(a, b)+\tilde{L} i_{1,1}(b, a)=\tilde{L} i_{1}(a) \tilde{L} i_{1}(b)-\tilde{L} i_{2}(a b), \\
\tilde{L} i_{1,1}(a, b)+\tilde{L} i_{1,1}\left(a^{-1}, a b\right)=\tilde{L} i_{1}(a b) \tilde{L} i_{1}(b)
\end{gathered}
$$


The distribution relations: For any $n \mid N$ one has

$$
\tilde{L} i_{1,1}\left(a_{1}, a_{2}\right)=\sum_{b_{i}^{n}=a_{i}} \tilde{L} i_{1,1}\left(b_{1}, b_{2}\right), \quad \tilde{L} i_{2}(a)=n \cdot \sum_{b^{n}=a} \tilde{L} i_{2}(b) .
$$

Let $\Delta_{12}$ be the group of order 12 given by generators $\sigma_{1}, \sigma_{2}$ subject to the relations $\sigma_{1}^{2}=\sigma_{2}^{2}=1,\left(\sigma_{1} \sigma_{2}\right)^{6}=1$. This group acts on the space of the depth two, weight two polylogarithms as follows:

$$
\begin{gathered}
\sigma_{1}: \tilde{L} i_{1,1}(a, b) \longmapsto-\tilde{L} i_{1,1}\left(a^{-1}, a b\right)+\tilde{L} i_{1}(a b) \tilde{L} i_{1}(b), \\
\sigma_{2}: \tilde{L} i_{1,1}(a, b) \longmapsto-\tilde{L} i_{1,1}(b, a)+\tilde{L} i_{1}(a) \tilde{L} i_{1}(b)-\tilde{L} i_{2}(a b) .
\end{gathered}
$$

The action on the depth one polylogarithms is trivial. The relations (9) are just the symmetry under the action of $\Delta_{12}$. (Compare this with the Bass theorem on cyclotomic units.)

Remark. Consider slightly modified functions

$$
\begin{gathered}
L_{1,1}^{\prime}(x, y):=L i_{1,1}(x, y)-\frac{1}{2} \log (1-x y) \cdot \log \frac{(1-y) x}{1-x}-\frac{1}{2} \log (1-x) \cdot \log (1-y) \\
L_{2}^{\prime}(x):=L i_{2}(x)-\frac{1}{2} \log (1-x) \cdot \log x
\end{gathered}
$$

Then we rid off products of logarithms from the symmetry relations (9)

$$
\tilde{L}_{1,1}^{\prime}(x, y)+\tilde{L}_{1,1}^{\prime}(y, x)+\tilde{L}_{2}^{\prime}(x y)=0, \quad \tilde{L}_{1,1}^{\prime}(x, y)+\tilde{L}_{1,1}^{\prime}\left(x^{-1}, x y\right)=0 .
$$

Definition 3.2. The subgroup $\mathcal{C}(N)_{2} \subset \mathcal{H}_{2} /\left(\mathcal{H}_{1}\right)^{2}$ is generated by the 2 -framed Hodge-Tate structures $\tilde{L} i_{1,1}(a, b), a^{N}=b^{N}=1$.

Thanks to (9), (10) we know that $\tilde{L} i_{2}(a) \in \mathcal{C}(N)_{2}$. Let $\mathcal{C}(N)_{2}^{(1)} \subset \mathcal{C}(N)_{2}$ be the subgroup generated by the motivic dilogarithms at $N$-th roots of unity, i.e. by $\tilde{L} i_{2}(a), a^{N}=1$. Set $\bar{C}(N)_{2}:=\mathcal{C}(N)_{2} / C(N)_{2}^{(1)}$

Definition 3.3. Let $a, b, c$ be $N$-th roots of unity such that $a b c=1$. Denote by $\{a, b, c\}$ be the projection of $\tilde{L} i_{1,1}(a, b)$ to $\overline{\mathcal{C}}(N)_{2}$.

The symmetry under the action of the group $\Delta_{12}$ now looks neater:

$$
\{a, b, c\}=-\{b, a, c\}, \quad\{a, b, c\}=-\{a, c, b\}, \quad\{a, b, c\}=\left\{a^{-1}, b^{-1}, c^{-1}\right\} .
$$

In particular $\Delta_{12}=S_{3} \times \mathbb{Z} / 2 \mathbb{Z}$. 
2. The double logarithm complex. Let $C_{N}^{*}$ be the subgroup of $\mathbb{C}^{*}$ generated by the elements $1-\zeta_{N}^{\alpha}$ where $\zeta_{N}$ is a primitive root of unity.

Lemma 3.4. $\quad$ a) Modulo $N$-torsion one has

$$
\mathcal{C}(N)_{2}^{(1)} \subset \operatorname{Ker} \delta, \quad \delta\left(\mathcal{C}(N)_{2}\right) \subset \Lambda^{2} C_{N}^{*} \cdot \mathbb{Z}\left[\frac{1}{N}\right] .
$$

b) $\mathcal{C}(N)_{2}^{(1)}=K_{3}^{\text {ind }}\left(\mathbb{Z}\left[\zeta_{N}\right]\right)$ modulo torsion.

c) If $N=p$ is a prime then modulo p-torsion $\delta\left(\mathcal{C}(p)_{2}\right) \subset \Lambda^{2} C_{p}$.

Proof. a) Since $\zeta_{N}$ is an $N$-torsion element in $\mathbb{C}^{*}$ we get

$$
\delta \tilde{L}_{2}\left(\zeta_{N}^{\alpha}\right)=\tilde{L}_{1}\left(\zeta_{N}^{\alpha}\right) \wedge \zeta_{N}^{\alpha}=0 \text { modulo } N \text {-torsion }
$$

Using formula $(7)$ for $\Delta^{\prime} \tilde{L} i_{1,1}(x, y)$ when $x, y$ are $N$-th roots of unity we get modulo $N$-torsion

$$
\begin{aligned}
\delta\{a, b, c\} & =-(1-a) \wedge(1-b)-(1-b) \wedge(1-c)-(1-c) \wedge(1-a) \\
& =\frac{1-a}{1-c} \wedge \frac{1-b}{1-c} .
\end{aligned}
$$

b) This is a reformulation of the well known result about the cyclotomic elements in $K_{3}\left(\mathbb{Z}\left[\zeta_{N}\right]\right)$.

c) Follows from (15) and the fact that $\frac{1-\zeta_{p}^{\alpha}}{1-\zeta_{p}^{\beta}}$ is a unit when $p$ is a prime.

So we get a complex defined modulo $N$-torsion

$$
\delta: \mathcal{C}(N)_{2} \longrightarrow \Lambda^{2} C_{N}^{*} \mathbb{Q} .
$$

Below we will compare it with Manin's complex for the modular curve $X_{1}(N)$.

3. Modular symbols and Manin's complex. Modular symbols. Let $x, y$ be two points on $P^{1}(\mathbb{Q})$ viewed as the boundary points of the hyperbolic plane $\mathcal{H}$. Let $\gamma_{x, y}$ be the geodesic connecting $x$ and $y$. Let $\Gamma \subset P S L_{2}(\mathbb{Z})$ be a subgroup of finite index. Set $Y_{\Gamma}:=\Gamma \backslash \mathcal{H}$. Let $\mathcal{H}^{*}:=\mathcal{H}^{*} \cup \mathbb{P}^{1}(\mathbb{Q})$. Let $X_{\Gamma}=$ $\Gamma \backslash \mathcal{H}^{*}$ be the compactification of $Y_{\Gamma}$ and $P_{\Gamma}:=X_{\Gamma} \backslash Y_{\Gamma}=\Gamma \backslash P^{1}(\mathbb{Q})$ the set of cusps. The projection of the geodesic $\gamma_{x, y}$ onto $X_{\Gamma}$ defines an element $\{x, y\} \in$ $H_{1}\left(X_{\Gamma}, P_{\Gamma} ; \mathbb{Z}\right)$, called a modular symbol. Let $X_{1}(N)_{\mathbb{C}}=\Gamma_{1}(N) \backslash \mathcal{H}^{*}$ be the modular curve of level $N . X_{1}(N)_{\mathbb{C}}$ is the set of complex points of an algebraic curve $X_{1}(N)$ which can be defined over $\mathbb{Q}$. So the complex conjugation acts on $X_{1}(N)(\mathbb{C})$. The projection $\Gamma_{1}(N) \backslash \mathcal{H}^{*} \longrightarrow X_{1}(N)(\mathbb{C})$ transforms the involution $z \rightarrow-\bar{z}$ of the hyperbolic plane to the complex conjugation on $X_{1}(N)(\mathbb{C})$.

Manin's complex. Let $\gamma$ be the vertical geodesic from 0 to $i \infty$ on the hyperbolic plane. For any $g \in P S L_{2}(\mathbb{Z})$ we get a geodesic $g \gamma$. Its projection onto $X_{1}(N)$ depends only on the coset $\Gamma_{1}(N) \cdot g$. Set

$$
E_{N}:=\frac{\left\{\langle\alpha, \beta\rangle \in(\mathbb{Z} / N \mathbb{Z})^{2} \mid \text { g.c.d. }(\alpha, \beta, N)=1\right\}}{<\alpha, \beta>\sim<-\alpha,-\beta\rangle} .
$$


The group $P S L_{2}(\mathbb{Z})$ acts from the right on the rows $\langle\alpha, \beta\rangle$ where $\alpha, \beta \in$ $(\mathbb{Z} / N \mathbb{Z})^{2} / \pm 1$. The set $E_{N}$ is the orbit of $<0,1>$, so $E_{N}=\Gamma_{1}(N) \backslash P S L_{2}(\mathbb{Z})$. Thus for any $\left\langle\alpha, \beta>\in E_{N}\right.$ we get a geodesic $\xi(\alpha, \beta)$ on $X_{1}(N)$.

Set

$$
\sigma=\left(\begin{array}{cc}
0 & -1 \\
1 & 0
\end{array}\right) \quad \tau=\left(\begin{array}{cc}
0 & -1 \\
1 & -1
\end{array}\right) .
$$

Let $\left\langle\gamma, \tau \gamma, \tau^{2} \gamma\right\rangle$ be the geodesic triangle with the vertices $i \infty, 0,1$.

Consider the following cell decomposition of $X_{1}(N)$ :

0-cells: cusps on $X_{1}(N)$.

1-cells: geodesics $g \cdot \gamma$ on $X_{1}(N), g \in P S L_{2}(\mathbb{Z})$.

2-cells: the projections onto $X_{1}(N)$ of the triangles $g \cdot\left\langle\gamma, \tau \gamma, \tau^{2} \gamma\right\rangle$, where $g \in P S L_{2}(\mathbb{Z})$.

Set $C_{i}\left(X_{1}(N)\right):=\mathbb{Z}[i-$ cells $], \quad C_{i}^{\mathbb{Q}}\left(X_{1}(N)\right):=C_{i}\left(X_{1}(N)\right) \otimes \mathbb{Q}$.

We get the chain complex of this cell decomposition of $X_{1}(N)$ :

$$
C_{2}\left(X_{1}(N)\right) \stackrel{\partial}{\longrightarrow} C_{1}\left(X_{1}(N)\right) \stackrel{\partial}{\longrightarrow} C_{0}\left(X_{1}(N)\right) \text {. }
$$
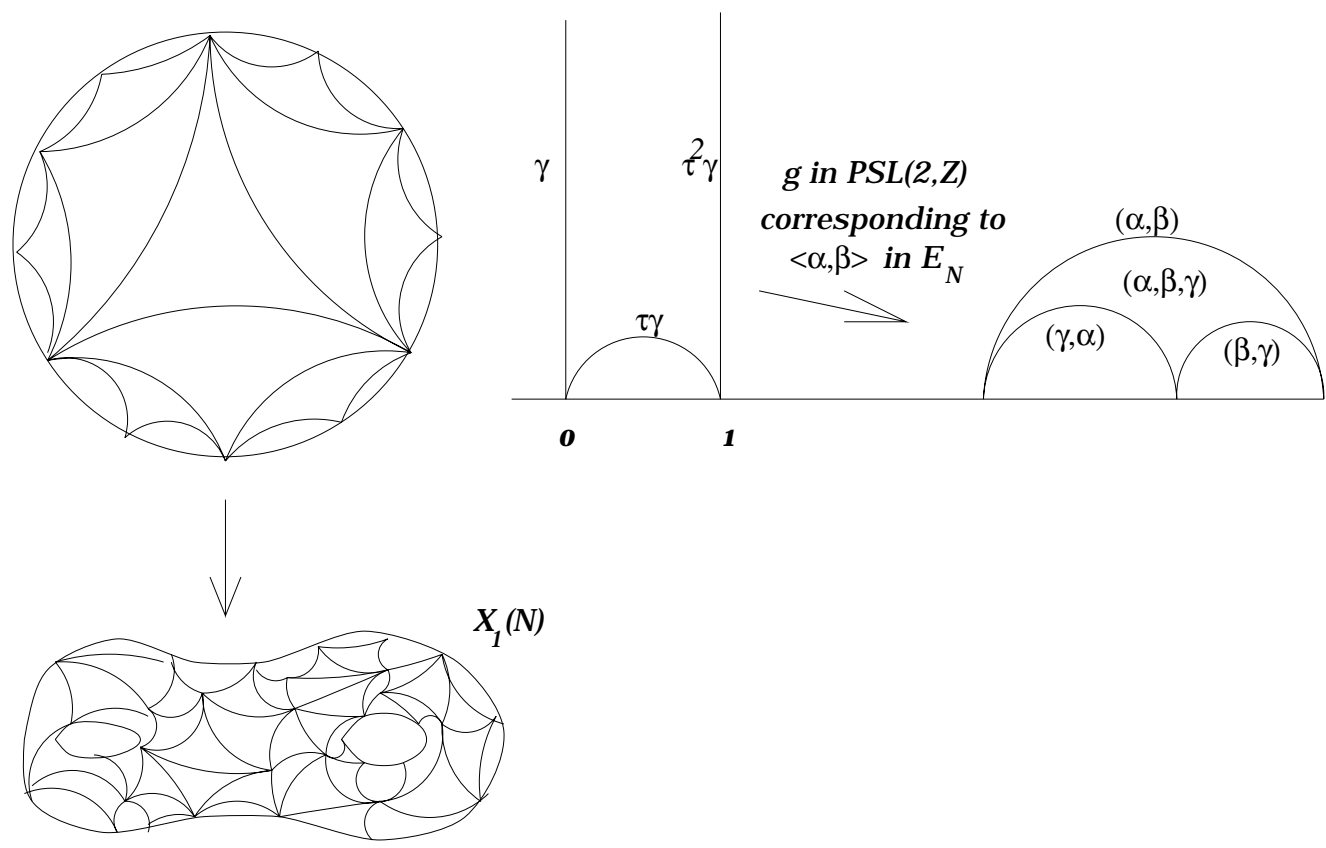

Let $C_{i}\left(X_{1}(N)\right)_{+}$be the space of coinvariants of the complex conjugation.

4. Main construction. Let $\alpha, \beta, \gamma \in \mathbb{Z} / N \mathbb{Z}$ and $\alpha+\beta+\gamma=0$. Choose $g \in P G L_{2}(\mathbb{Z})$ such that $\Gamma_{1}(N) \cdot g=<\alpha, \beta>$. Denote by $\xi(\alpha, \beta, \gamma)$ the oriented triangle $g \cdot\left\langle\gamma, \tau \gamma, \tau^{2} \gamma\right\rangle$. Its sides are the geodesics $\xi(\alpha, \beta), \xi(\beta, \gamma), \xi(\gamma, \alpha)$. Let $(\alpha, \beta, \gamma)$ be the generator of the group $C_{2}\left(X_{1}(N)\right)_{+}$corresponding to this 
triangle. Denote by $(\alpha, \beta)$ the element of the group $C_{1}\left(X_{1}(N)\right)_{+}$corresponding to the geodesic $\xi(\alpha, \beta)$. Then

$$
\partial(\alpha, \beta, \gamma)=(\alpha, \beta)+(\beta, \gamma)+(\gamma, \alpha)
$$

Theorem 3.5. a) Let $N>1$ and $\zeta_{N}:=e^{2 \pi i / N}$. Then modulo $N$-torsion there is a morphism of complexes

$$
\begin{array}{ccc}
C_{2}\left(X_{1}(N)\right)_{+} & \stackrel{\partial}{\longrightarrow} & C_{1}\left(X_{1}(N)\right)_{+} \\
g_{2} \downarrow & g_{1} \downarrow \\
\mathcal{C}(N)_{2} & \stackrel{\delta}{\longrightarrow} & \Lambda^{2} C_{N}^{*}
\end{array}
$$

given on the generators by

$$
\begin{gathered}
g_{1}:(\alpha, \beta, \gamma) \longmapsto-\left\{\zeta_{N}^{\alpha}, \zeta_{N}^{\beta}, \zeta_{N}^{\gamma}\right\}, \quad \alpha+\beta+\gamma=0 \\
g_{2}:(\alpha, \beta) \longmapsto\left(1-\zeta_{N}^{\alpha}\right) \wedge\left(1-\zeta_{N}^{\beta}\right), \quad(0, \beta) \longmapsto 0 .
\end{gathered}
$$

b) It is surjective if $N=p^{a}$ where $p$ is a prime.

Proof. The map $g_{1}$ is obviously surjective for any $N$.

Proposition 3.6. $g_{2}$ is a well defined homomorphism.

Proof of the proposition (3.6). The group $S_{3} \times \mathbb{Z} / 2 \mathbb{Z}$ acts on the generators $(\alpha, \beta, \gamma)$ of the group $C_{2}\left(X_{1}(N)\right)_{+}$. Namely, $\sigma \in S_{3}$ acts by a permutation and the nontrivial element of $\mathbb{Z} / 2 \mathbb{Z}$ by $(\alpha, \beta, \gamma) \longmapsto(-\alpha,-\beta,-\gamma)$.

Lemma 3.7. One has

$$
(\alpha, \beta, \gamma)=(-\alpha,-\beta,-\gamma), \quad(\alpha, \beta, \gamma)=(\gamma, \alpha, \beta), \quad(\alpha, \beta, \gamma)=-(\beta, \alpha, \gamma) .
$$

Proof. The first equality follows from the definition of $E_{N}$. The second is true since the triangles $\left\langle g \cdot \gamma, g \tau \cdot \gamma, g \tau^{2} \cdot \gamma>\right.$ and $\left\langle g \tau \cdot \gamma, g \tau^{2} \cdot \gamma, g \cdot \gamma>\right.$ coinside. The third is valid thanks to formula (19) in the following lemma

Lemma 3.8. a) The complex conjugation acts on the geodesic $\xi(\alpha, \beta)$ by

$$
\xi(\alpha, \beta) \longmapsto \xi(-\alpha, \beta,)
$$

and on the generators of the group $C_{2}\left(X_{1}(N)\right)$ by

$$
(\alpha, \beta, \gamma) \longmapsto-(\beta, \alpha, \gamma)
$$

b) One has $(\alpha, \beta)=-(\beta,-\alpha)$. 
Proof. a) Let $g=\left(\begin{array}{ll}a & b \\ c & d\end{array}\right)$. Then $g \cdot\{i \infty, 0\}=\left\{\frac{a}{c}, \frac{b}{d}\right\}=\xi(c, d)$. The involution $z \rightarrow-\bar{z}$ sends the modular symbol $\left\{\frac{a}{c}, \frac{b}{d}\right\}$ to $\left\{-\frac{a}{c},-\frac{b}{d}\right\}$. On the other hand

$$
\left\{-\frac{a}{c},-\frac{b}{d}\right\}=\left(\begin{array}{cc}
a & -b \\
-c & d
\end{array}\right)\{i \infty, 0\}=\xi(-c, d),
$$

and we get (18). Notice $g \tau=\left(\begin{array}{cc}b & -a-b \\ d & -c-d\end{array}\right), \quad g \tau^{2}=\left(\begin{array}{ll}-a-b & b \\ -c-d & d\end{array}\right)$. So the element $g=\left(\begin{array}{ll}a & b \\ c & d\end{array}\right)$ transforms the triangle $\left\langle\gamma, \tau \gamma, \tau^{2} \gamma>\right.$ to the geodesic triangle $<\xi(c, d), \xi(d,-c-d), \xi(-c-d, c)>$. Under the complex conjugation it goes to the geodesic triangle $<\xi(-c, d), \xi(-d,-c-d), \xi(c+d, c)>$, which coinsides with the triangle $<\xi(d, c), \xi(-c-d, d), \xi(c,-c-d)>$ but has a different orientation.

b) Indeed, $\sigma \gamma$ is the geodesic from $i \infty$ to 0 and $\Gamma_{1}(N) \cdot g \sigma=(\beta,-\alpha)$. The lemma is proved.

The stabilizer of the triangle $\left\langle\gamma, \tau \gamma, \tau^{2} \gamma\right\rangle$ in the group $P G L_{2}(\mathbb{Z})$ is the group $S_{3}$. So the only relations between the generators $(\alpha, \beta, \gamma)$ are those provided by the action of the group $S_{3} \times \mathbb{Z} / 2 \mathbb{Z}$. Under the map $g_{2}$ they go precisely to the symmetry relations (14). So $g_{2}$ is well defined. Proposition (3.6) is proved.

All relations between the generators $(\alpha, \beta)$ of $C_{1}\left(X_{1}(p)\right)_{+}$are given by (18) and lemma (3.8b). So $g_{1}$ is well defined.

Looking at the diagram

$$
\begin{array}{ccc}
(\alpha, \beta, \gamma) & \stackrel{\partial}{\longrightarrow} & (\alpha, \beta)+(\beta, \gamma)+(\gamma, \alpha) \\
g_{2} \downarrow & & g_{1} \downarrow \\
-\left\{\zeta_{N}^{\alpha}, \zeta_{N}^{\beta}, \zeta_{N}^{\gamma}\right\} \stackrel{\delta}{\longrightarrow} \quad\left(1-\zeta_{N}^{\alpha}\right) \wedge & \left(1-\zeta_{N}^{\beta}\right)+\left(1-\zeta_{N}^{\beta}\right) \wedge\left(1-\zeta_{N}^{\gamma}\right) \\
& & \left(1-\zeta_{N}^{\gamma}\right) \wedge\left(1-\zeta_{N}^{\alpha}\right)
\end{array}
$$

and comparing formula (15) for $\delta$ with formula (17) for $\partial$ we see the commutativity for $\alpha \beta \gamma \neq 0$. Notice that $(0, \beta,-\beta)=0$ and $\left(1-\zeta_{N}^{\beta}\right) \wedge\left(1-\zeta_{N}^{-\beta}\right)=0$ modulo $N$-torsion. So the diagram is commutative. Theorem (3.5a) is proved.

5. The case when the level $N=p$ is a prime. We will construct a subcomplex $\tilde{C}_{\bullet}\left(X_{1}(p)\right) \subset C_{\bullet}\left(X_{1}(p)\right)$. For this we need to recall some facts.

The boundary of Manin's symbols. The cusps of $X_{1}(N)$ are parametrized by the orbits of the unipotent group $\Gamma_{\infty}:=\left(\begin{array}{ll}1 & * \\ 0 & 1\end{array}\right)$ on the set $E_{N}$. If $N=p$ is a prime they are the orbits of the elements $\langle 0, \beta\rangle$ and $\langle\alpha, 0\rangle$ where $\alpha \neq 0, \beta \neq 0$. We will denote these orbits by $[0, \beta]$ and $[\alpha, 0]$.

The boundary of Manin's symbols looks as follows:

$$
\partial(\alpha, \beta)=[0, \beta]-[0, \alpha] \quad \text { if } \alpha \neq 0, \beta \neq 0
$$




$$
\partial(0, \beta)=-\partial(\beta, 0)=[0, \beta]-[\beta, 0] \quad \text { if } \beta \neq 0 ;
$$

From this and lemma 3.8 we see that the complex conjugation acts as the identity map on the cusps of $X_{1}(p)$.

There are just two cusps on $X_{0}(p)$ called the $\infty$ and 0 cusps. They are the images of $i \infty$ and 0 under the projection $\mathcal{H}^{*} \rightarrow X_{0}(p)$. The covering $X_{1}(p) \rightarrow$ $X_{0}(p)$ is unramified of degree $(p-1) / 2$. The cusps on $X_{1}(p)$ lying over the $\infty$ cusp on $X_{0}(p)$ are called the $\infty$-cusps. They are canonically identified with the set of $p$-th roots of unity different from 1 considered modulo inversion (i.e. $\zeta$ and $\zeta^{-1}$ correspond to the same cusp). If we think about the cusps as of the isomorphism classes of pairs,

\{ a generalized elliptic curve of Deligne-Rappoport, a point of order $p$ on it such that the subgroup scheme it generates meets every irreducible component of each geometrical fiber $\}$,

then the $\infty$-cusps correspond to the standard Neron polygon with one side and the choice of a generator of its unique subgroup of order $p$. In our parametrization this is the cusps $[0, \beta]$. By definition $\tilde{C}_{0}\left(X_{1}(p)\right)$ is the free abelian group generated by the $\infty$-cusps. So there is a canonical isomorphism modulo $p$-torsion

$$
g_{0}: \tilde{C}_{0}\left(X_{1}(p)\right) \longrightarrow C_{p}^{*}, \quad[0, \beta] \longmapsto 1-\zeta_{p}^{\beta}
$$

group $\tilde{C}_{1}\left(X_{1}(p)\right)$ is generated by the geodesics $\xi(\alpha, \beta)$ connecting the $\infty$-cusps i.e. $\alpha \beta \neq 0$. The group $\tilde{C}_{2}\left(X_{1}(p)\right)$ is generated by the geodesic triangles $(\alpha, \beta, \gamma)$ such that $\alpha \beta \gamma \neq 0$. These groups form a subcomplex $\tilde{C}_{\bullet}\left(X_{1}(p)\right)$ of Manin's complex. Its + part is canonically quasiisomorphic to the + part of Manin's complex. Indeed, each 0-cusp is a boundary of just one edge in Manin's complex connecting it with an $\infty$-cusp. $([0, \beta]$ is connected with $[\beta, 0])$. Notice that $(0, \beta,-\beta)=0$ in $C_{2}\left(X_{1}(p)\right)_{+}$.

over $\infty$ with the $p$-th roots of unity modulo

Consider a homomorphism

$$
\delta^{\prime}: \Lambda^{2} C_{p}^{*} \longrightarrow C_{p}^{*}, \quad\left(1-\zeta_{p}^{\alpha}\right) \wedge\left(1-\zeta_{p}^{\beta}\right) \longmapsto \frac{1-\zeta_{p}^{\beta}}{1-\zeta_{p}^{\alpha}}
$$

The kernel of this map modulo $p$-torsion is the group $\Lambda^{2} C_{p}$.

Theorem 3.9. a) There is a canonical isomorphism of complexes modulo ptorsion

$$
\begin{array}{ccccc}
\tilde{C}_{2}\left(X_{1}(p)\right)_{+} & \longrightarrow & \tilde{C}_{1}\left(X_{1}(p)\right)_{+} & \longrightarrow & \tilde{C}_{0}\left(X_{1}(p)\right)_{+} \\
g_{2} \downarrow & g_{1} \downarrow & & g_{0} \downarrow \\
\overline{\mathcal{C}}(p)_{2} & \stackrel{\delta}{\longrightarrow} & \Lambda^{2} C_{p}^{*} & \stackrel{\delta^{\prime}}{\longrightarrow} & C_{p}^{*}
\end{array}
$$

b) It transmits the action of the Galois group of the covering $X_{1}(p) \rightarrow X_{0}(p)$ on the complex $\tilde{C}_{\bullet}\left(X_{1}(p)\right)$ to the action of $\operatorname{Gal}\left(\mathbb{Q}\left(\zeta_{p}\right) / \mathbb{Q}\right)$ on the bottom complex. 
Remark. In the definition of Manin's complex for $X_{1}(N)$ we have already choosen the generator $e^{2 \pi i / N}$ of the group of $N$-th roots of unity.

Proof. The only relations modulo $p$-torsion in $C_{p}^{*}$ are $1-\zeta_{p}^{\alpha}=1-\zeta_{p}^{-\alpha}$. Thanks to this, lemma (3.8b) and formula (18), the map $g_{1}$ is an isomorphism.

The complex conjugation acts on $H_{2}\left(X_{1}(p), \mathbb{Q}\right)$ with the eigenvalue -1 . This means that the restriction of $\partial$ to $C_{2}\left(X_{1}(p)\right)_{+}$is injective. Since the diagram is commutative and $g_{1}$ is an isomorphism, we conclude that $g_{2}$ is injective. So it is an isomorphism by theorem (3.5). The theorem is proved.

6. Corollaries. Set $Z_{1}\left(X_{1}(p)\right)_{+}:=\operatorname{Ker}\left(C_{1}\left(X_{1}(p)\right)_{+} \stackrel{\partial}{\longrightarrow} C_{0}\left(X_{1}(p)\right)_{+}\right)$.

Corollary 3.10. a) There is an isomorphism of complexes modulo p-torsion

$$
\begin{array}{ccc}
C_{2}\left(X_{1}(p)\right)_{+} & \stackrel{\partial}{\longrightarrow} & Z_{1}\left(X_{1}(p)\right)_{+} \\
g_{2} \downarrow & & \downarrow g_{1} \\
\overline{\mathcal{C}}(p)_{2} & \stackrel{\delta}{\longrightarrow} & \Lambda^{2} C_{p}
\end{array}
$$

b) $\operatorname{dim} \overline{\mathcal{C}}(p)_{2}=\frac{(p-1)(p-5)}{12}$.

Proof. a) follows from theorem (3.9). To check b) notice that $r k \Lambda^{2} C_{p}=$ $\frac{(p-3)(p-5)}{8}$ and $\operatorname{dim} S_{2}^{0}\left(\Gamma_{1}(p)\right)=1+\frac{p^{2}-1}{24}-\frac{p-1}{2}$.

Example. $p=13$, so $\operatorname{dim} \overline{\mathcal{C}}(13)_{2}=8, \operatorname{dim} S_{2}^{0} \Gamma_{1}(13)=2$.

\section{Corollary 3.11.}

$$
\begin{gathered}
\operatorname{Ker}\left(\mathcal{C}_{2}(p) \stackrel{\delta}{\longrightarrow} \Lambda^{2} C_{p}\right) \otimes \mathbb{Q}=K_{3}\left(\mathbb{Z}\left[\zeta_{p}\right]\right) \otimes \mathbb{Q} \\
\operatorname{Coker}\left(\mathcal{C}_{2}(p) \stackrel{\delta}{\longrightarrow} \Lambda^{2} C_{p}\right)=H_{1}\left(X_{1}(p), \mathbb{Z}\right)_{+} \quad \text { modulo p-torsion } .
\end{gathered}
$$

The analog of the Bass theorem on the cyclotomic units is

Theorem 3.12. All relations between the motivic depth two polylogarithms at p-th roots of unity are given by the symmetry relations (8) and (9).

The corollary and the theorem follow immediately from theorem (3.9). 
7. Motivic picture. Let $\mathcal{M}_{T}(S)$ be the abelian category of mixed Tate motives over one dimensional arithmetic scheme $S$. The key property is the fundamental Beilinson formula

$$
\operatorname{Ext}_{\mathcal{M}_{T}(S)}^{1}(\mathbb{Q}(0), \mathbb{Q}(n))=K_{2 n-1}(S) \otimes \mathbb{Q}, \quad n \geq 1
$$

and higher Ext's vanish. The corresponding triangulated category was recentely defined by Voevodsky, however the key formula (??) is not available yet. So we can define an object of $\mathcal{M}_{T}(S)$, but can not afford to use the whole formalism. In this section we will assume the formalism, so the results are conditional.

We will say that an equivalence class of $n$-framed mixed Tate motives is defined over $S$ if one can find a representative in the equivalence class defined over $S$.

Theorem 3.13. Let us assume (??). Suppose that $a_{1}^{p}=a_{2}^{p}=1$ and $p$ is a prime number. Then there exist $M \in \mathbb{Z}$ such that the equivalence class of the 2-framed mixed Tate motive $M \cdot \tilde{L} i_{1,1}\left(a_{1}, a_{2}\right)$ is defined over $S_{p}:=$ Spec $\mathbb{Z}\left[\zeta_{p}\right]$.

However, we do not get all 2-framed mixed Tate motives over $S_{p}$ this way! Namely, let $\mathcal{L}(p)$. be the Lie coalgebra of the category of mixed Tate motives over $S_{p}$. Then it follows from the Tannakian formalism (see s. 2.4) that $H_{(n)}^{i}\left(\mathcal{L}(p)_{\bullet}\right)=\operatorname{Ext}_{\mathcal{M}_{T}\left(S_{p}\right)}(\mathbb{Q}(0), \mathbb{Q}(n))$ where $H_{(n)}^{i}$ is the degree $n$ part of $H^{i}$. So by (??) one has $\mathcal{L}(p)_{1}=C_{p} \otimes \mathbb{Q}$,

$$
\begin{aligned}
\operatorname{Coker}\left(\mathcal{L}(p)_{2} \rightarrow \Lambda^{2} \mathcal{L}(p)_{1}\right) & =\operatorname{Ext}_{\mathcal{M}_{T}\left(S_{p}\right)}^{2}(\mathbb{Q}(0), \mathbb{Q}(2)) \\
& =K_{2}\left(\mathbb{Z}\left[\zeta_{p}\right]\right) \otimes \mathbb{Q}=0,
\end{aligned}
$$

$\operatorname{Ker}\left(\mathcal{L}(p)_{2} \rightarrow \Lambda^{2} \mathcal{L}(p)_{1}\right)=\operatorname{Ext}_{\mathcal{M}_{T}\left(S_{p}\right)}^{1}(\mathbb{Q}(0), \mathbb{Q}(2))=K_{3}\left(\mathbb{Z}\left[\zeta_{p}\right]\right) \otimes \mathbb{Q} \subset C(p)_{2} \otimes \mathbb{Q}$.

Theorem (??) shows that $\mathcal{C}(p)_{2} \otimes \mathbb{Q} \subset \mathcal{L}(p)_{2}$. So $\delta$ provides an isomorphism

$$
\frac{\mathcal{L}(p)_{2}}{\mathcal{C}(p)_{2} \otimes \mathbb{Q}}=\frac{\Lambda^{2} C_{p} \otimes \mathbb{Q}}{\delta\left(\mathcal{C}(p)_{2}\right)}
$$

Thanks to (??) the right hand side is isomorphic to $H_{1}\left(X_{1}(p), \mathbb{Q}\right)_{+}$. So we get

Theorem 3.14. Let us assume (??). Then there exists a canonical isomorpism

$$
\frac{\mathcal{L}(p)_{2}}{\mathcal{C}(p)_{2} \otimes \mathbb{Q}}=H_{1}\left(X_{1}(p), \mathbb{Q}\right)_{+}
$$

Proof of theorem (??). It is easy to show that $\tilde{L} i_{1,1}\left(a_{1}, a_{2}\right)$ is defined over $S_{p}^{*}:=$ $\operatorname{Spec} \mathbb{Z}\left[\zeta_{p}\right]\left[\frac{1}{p}\right]$. One has $\delta \tilde{L} i_{1,1}\left(a_{1}, a_{2}\right) \in \Lambda^{2} C_{p}$ modulo $p$-torsion. So by (??) there exists a 2 -framed mixed Tate motive $X$ over $S_{p}$ such that $M \cdot \delta(X-$ $\left.\tilde{L} i_{1,1}\left(a_{1}, a_{2}\right)\right)=0$ for certain $M \in \mathbb{Z}$. So $M\left(X-\tilde{L} i_{1,1}\left(a_{1}, a_{2}\right)\right)$ represents a class in $\operatorname{Ext}_{\mathcal{M}_{T}\left(S_{p}^{*}\right)}^{1}(\mathbb{Q}(0), \mathbb{Q}(2))$, which is given by $\sum n_{a} \tilde{L} i_{2}\left(\zeta_{p}^{a}\right)$ and thus defined over $S_{p}$. 


\section{Multiple polylogarithms at roots of unity: fragments of the picture}

\section{Motivic point of view and its consequences.}

Theorem 4.1. $\quad$ a) Suppose that $a_{i}^{p}=1$. Then $\tilde{L} i_{n_{1}, \ldots, n_{l}}\left(a_{1}, \ldots, a_{l}\right)$ is a mixed Tate motive over the scheme $S_{p}^{*}:=\operatorname{Spec}\left(\mathbb{Z}[x] /\left(x^{p}-1\right)\left[\frac{1}{p}\right]\right)$.

b) In particular $\tilde{\zeta}\left(n_{1}, \ldots, n_{l}\right):=\tilde{L} i_{n_{1}, \ldots, n_{l}}(1, \ldots, 1)$ is a $w$-framed mixed Tate motive over Spec $\mathbb{Z}$.

Let $L(\mathbb{Z})$ • be the free graded Lie algebra generated by elements $e_{(2 n+1)}$ of degree $-(2 n+1)(n \geq 1)$ and $U L(\mathbb{Z})^{*}$ be the dual to its universal enveloping algebra (graded by positive integers). Let $f_{3}, f_{5}, \ldots$ be the functionals on the vector space generated by the vectors $e_{3}, e_{5}, \ldots$ such that $\left\langle f_{i}, e_{j}\right\rangle=0$. Then $U L(\mathbb{Z})_{\bullet}^{*}$ is isomorphic to the space of noncomutative polynomials on $f_{2 n+1}$ with the shuffle product.

Let $Z_{w}$ be the $\mathbb{Q}$-vector space generated by the numbers $\zeta\left(n_{1}, \ldots, n_{l}\right)$ of weight $w$. Then $Z_{\bullet}:=\sum Z_{w}$ is obviously an algebra.

Conjecture 4.2. $\quad$ a) The weight provides a grading on the algebra $Z_{\bullet}$.

b) One has an isomorphism of graded algebras

$$
Z_{\bullet} \quad=\quad \mathbb{Q}\left[\pi^{2}\right] \otimes U L(\mathbb{Z})_{\bullet}^{*} \quad \operatorname{deg}\left(\pi^{2}\right)=2 .
$$

In particular $Z_{\bullet}$ should have a natural structure of a graded Hopf algebra. Part a) means that relations between $\zeta$ 's of different weight, like $\zeta(5)=\lambda \cdot \zeta(7)$ where $\lambda \in \mathbb{Q}$ are impossible.

Let $\mathcal{F}(2,3)$ be the free graded Lie algebra generated by 2 elements of degree -2 and -3 . $U \mathcal{F}(2,3)^{*}$ is isomorphic as a graded vector space to the space of noncommutative polynomials in 2 variables $p$ and $g_{3}$ of degrees 2 and 3 . There is canonical isomorphism of graded vector spaces $\mathbb{Q}\left[\pi^{2}\right] \otimes U L_{\bullet}(\mathbb{Z})^{*}=U \mathcal{F}(2,3)^{*}$. The rule is clear from the pattern $\left(\pi^{2}\right)^{3} f_{3}\left(f_{7}\right)^{3}\left(f_{5}\right)^{2} \longrightarrow p^{3} g_{3}\left(g_{3} p^{2}\right)^{3}\left(g_{3} p\right)^{2}$. In particular if $d_{k}:=\operatorname{dim} Z_{k}$ then one should have $d_{k}=d_{k-2}+d_{k-3}$. Computer calculations of D.Zagier lead to this formula for $k \leq 12$. Just recently much more extensive calculations, also confirming the formula above, were made by D. Broadhurst.

Remark. Let $\pi_{1}^{(l)}\left(\mathbb{P}^{1} \backslash\{0,1, \infty\}\right)$ be the $l$-adic completion of the fundamental group. One has canonical homomorphism

$$
\varphi^{l}: \operatorname{Gal}(\overline{\mathbb{Q}} / \mathbb{Q}) \longrightarrow \operatorname{Out} \pi_{1}^{(l)}\left(\mathbb{P}^{1} \backslash\{0,1, \infty\}\right) .
$$

It was studied by Deligne, Ihara and others (see the beautiful talk [Ih] delivered by Ihara in ICM-90, Kyoto and references there). Conjecture (??) is closely related to some conjectures/questions of Deligne $[\mathrm{D}]$ and Ihara about the image of the map (??) and Drinfeld [Dr] about the structure of the GrothendiekTeichmuller group. Namely, conjecture ?? means that the image of the map (??) should be as big as possible, i.e. the field stabilized by $\operatorname{Ker} \varphi^{l}$ is the maximal 
pro- $l$ extension of the $l^{\infty}$-cyclotomic extension of $\mathbb{Q}$ unramified outside $l$, and that the Lie algebra of the Grothendiek-Teichmuller group should be isomorphic to $L(\mathbb{Z})$ •.

Theorem 4.3. Let us assume (??). Then

$$
\operatorname{dim} Z_{k} \leq \text { the dimension of the degree } k \text { part of } U \mathcal{F}(2,3)^{*} .
$$

This is a consequence of the part b) of the previous theorem.

2. On a generalization of conjecture ?? to multiple polylogarithms at roots of unity. Let $Z(N)_{w}$ be the space of $\mathbb{Q}$-span of the numbers

$$
\bar{L} i_{n_{1}, \ldots, n_{l}}\left(\zeta_{N}^{\alpha_{1}}, \ldots, \zeta_{N}^{\alpha_{m}}\right):=\frac{1}{(2 \pi i)^{w}} L i_{n_{1}, \ldots, n_{l}}\left(\zeta_{N}^{\alpha_{1}}, \ldots, \zeta_{N}^{\alpha_{m}}\right)
$$

Then $Z(N)_{\bullet}:=\sum Z(N)_{w}$ is a bifiltered by the weight and by the depth.

Let $L\left(S_{N}^{*}\right)$ • be the free graded Lie algebra generated by the $\mathbb{Q}$-vector spaces $K_{2 n-1}\left(S_{N}^{*}\right) \otimes \mathbb{Q}$ in degrees $n \geq 1$. It is isomorphic to the Lie algebra of the (unipotent part of) the Galois group of the category $\mathcal{M}_{T}\left(S_{N}^{*}\right)$. This follows from (??) and the Tannakian formalism (see s.2.4).

Conjecture 4.4. a) There exists a quotient $C(N)$. of the graded Lie algebra $L\left(S_{N}^{*}\right)$ • such that one has an isomorphism of filtered (by the weight on the left and by the degree on the right) graded spaces

$$
Z(N)_{>0} / Z(N)_{>0}^{2}=C(N)_{\bullet}^{*}
$$

One can deduce from theorem ?? and the motivic formalism the upper bound: $\operatorname{dim} Z(N)_{w} /$ products $\leq \operatorname{dim} L\left(S_{N}^{*}\right)_{w}$.

Example. Using Borel's computation of $K$-groups of number fields [Bo] we get the following explicit description of the Lie algebra $L\left(S_{p}^{*}\right)$ •.

a) If $p=1$ then $L(\operatorname{Spec} \mathbb{Z}) \bullet$ is a free graded Lie algebra with one generator in all odd degrees starting from 3 , (corresponding to $\bar{\zeta}(3), \bar{\zeta}(5), \ldots$ ).

b) Let $p=2$. Then $L\left(S_{2}^{*}\right) \bullet$ is a free graded Lie algebra with one generator in each odd degree (corresponding to $\left.(2 \pi i)^{-1} \log 2, \bar{\zeta}(3), \bar{\zeta}(5), \ldots\right)$.

c) Let $p>2$. Then $r k K_{2 n-1}\left(S_{p}^{*}\right)=\frac{p-1}{2}, \quad n \geq 1$ and thus $L_{\bullet}\left(S_{p}^{*}\right)$ is a free Lie algebra with $(p-1) / 2$ generators in each positive degree (related to $(2 \pi i)^{-1} \log \left(1-\zeta_{p}^{a}\right), \bar{L} i_{2}\left(\zeta_{p}^{a}\right), \bar{L} i_{2}\left(\zeta_{p}^{a}\right), \ldots$, where $\left.1 \leq a \leq(p-1) / 2\right)$.

The conjecture ?? claims that this bound is exact for $p=1$. Nothing like that could happen for any prime $p>7$. Indeed, the only weight two multiple polylogarithms are the dilogarithm and the double logarithm, so by (??) $H_{(2)}^{2}\left(C(p)_{\bullet}\right)=H_{1}\left(X_{1}(p), \mathbb{Q}\right)_{+}$. Therefore $H_{1}\left(X_{1}(p), \mathbb{Q}\right)_{+}$is isomorphic to the space of the 2 -framed mixed motives over Spec $\mathbb{Z}\left[\zeta_{p}\right]$ "missed" by the multiple polylogarithms construction. So in general $C(N)$ • is not free and thus smaller then $L\left(S_{N}^{*}\right)$. . However recent computer calculations of $\mathrm{D}$. Broadhurst [Br] show that for $p=2$ the upper bound is exact for the weights $\leq 10$. 
3. Explicit description of the depth two Hopf algebra of the multiple polylogarithms. The framed mixed Hodge structures corresponding to multiple polylogarithms form a Hopf algebra. Its restriction to $N$-th roots of unity is the Hopf algebra $U(\mathcal{C}(N) \bullet)^{\vee}$ whose structure miraculously related with $G L(\mathbb{Z})$. The associated graded quotient with respect to the depth filtration of the Lie coalgebra $\mathcal{C}(N)$ • described explicitely in [G4]. Below we present the formulas in the first nontrivial case of the depth two polylogarithms. The general case will be treated in [G1] (see also [G3]).

Let $*$ be the product in the Hopf algebra. To simplify the formulas below, write $e^{x \cdot t}$ for $\exp \left(\tilde{L} i_{1}(1-x) \cdot t\right)$. Recall $\Delta^{\prime}(X):=\Delta(X)-(X \otimes 1+1 \otimes X)$.

Let us extend formally the definition of $\tilde{L} i_{n_{1}, n_{2}}(x, y)$ to the case when $n_{i} \in \mathbb{Z}$

$$
\begin{aligned}
\tilde{L} i_{n}(x) & =0, \quad \text { and } \quad \tilde{L} i_{m, n}(x, y)=0 \quad \text { if } \quad n \leq 0, \\
\tilde{L} i_{m, n}(x, y) & =-\tilde{L} i_{m+n}(x y) \quad \text { if } \quad m \leq 0 .
\end{aligned}
$$

Set

$$
\tilde{L}\left(x_{1}, x_{2} \mid t_{1}, t_{2}\right):=\sum_{n_{1}, n_{2} \in \mathbb{Z}} \tilde{L} i_{n_{1}, n_{2}}\left(x_{1}, x_{2}\right) t_{1}^{n_{1}-1} t_{2}^{n_{2}-1} .
$$

Theorem 4.5. The coproduct for the framed mixed Hodge structures related to multiple polylogarithms of depths 1 and 2 is given by the following formulas:

$$
\Delta^{\prime}: \tilde{L}\left(x_{1} \mid t_{1}\right) \longmapsto \tilde{L}\left(x_{1} \mid t_{1}\right) \otimes\left(e^{x_{1} \cdot t_{1}}-1\right)
$$

$$
\begin{aligned}
& \tilde{L}\left(x_{1}, x_{2} \mid t_{1}, t_{2}\right) \otimes\left(e^{x_{1} \cdot t_{1}+x_{2} \cdot t_{2}}-1\right)+\tilde{L}\left(x_{2} \mid t_{2}\right) \otimes e^{x_{2} \cdot t_{2}} * \tilde{L}\left(x_{1} \mid t_{1}\right) \\
& +\tilde{L}\left(x_{1} x_{2} \mid t_{1}\right) \otimes e^{x_{1} x_{2} \cdot t_{1}} * \tilde{L}\left(x_{2} \mid t_{2}-t_{1}\right)-\tilde{L}\left(x_{1} x_{2} \mid t_{2}\right) \otimes e^{x_{1} x_{2} \cdot t_{2}} * \tilde{L}\left(x_{1} \mid t_{1}-t_{2}\right) .
\end{aligned}
$$

For example

$$
\begin{gathered}
\Delta^{\prime}: \tilde{L} i_{2,1}\left(x_{1}, x_{2}\right) \longmapsto \tilde{L} i_{1,1}\left(x_{1}, x_{2}\right) \otimes x_{1}+\tilde{L} i_{1}\left(x_{2}\right) \otimes \tilde{L} i_{2}\left(x_{1}\right)+\tilde{L} i_{2}\left(x_{1} x_{2}\right) \otimes \tilde{L} i_{1}\left(x_{2}\right) \\
-\tilde{L} i_{1}\left(x_{1} x_{2}\right) \otimes\left(\tilde{L} i_{2}\left(x_{1}\right)+\tilde{L} i_{2}\left(x_{2}\right)-\tilde{L} i_{1}\left(x_{2}\right) *\left(x_{1} x_{2}\right)+\frac{x_{1}^{2}}{2}\right) .
\end{gathered}
$$

4. The motivic complex of the double $\zeta$ 's and cuspidal cohomology of $S L_{2}(\mathbb{Z})([\mathbf{G} \mathbf{3}])$. We define the framed Hodge-Tate structures $\tilde{\zeta}(1)$ and $\tilde{\zeta}(n, 1)$ (corresponding to the divergent series) as the limiting mixed Hodge structures for $L_{1}(x)$ and $L_{n, 1}(x, y)$ at $x=1$ and $x=y=1$. Then

$$
\tilde{\zeta}(1)=0, \quad \tilde{\zeta}(1,1)=0, \quad \tilde{\zeta}(n, 1)=-\sum_{i=2}^{n-1} \tilde{\zeta}(n-i, i)-\tilde{\zeta}(1, n) \quad(n>1) .
$$

For example $\tilde{\zeta}(3,1)=-\tilde{\zeta}(2,2)-2 \tilde{\zeta}(1,3)$. Setting $x_{1}=x_{2}=1$ in (??) we get $(28) \Delta^{\prime}: \tilde{\zeta}\left(t_{1}, t_{2}\right) \longmapsto \tilde{\zeta}\left(t_{2}\right) \otimes \tilde{\zeta}\left(t_{1}\right)+\tilde{\zeta}\left(t_{1}\right) \otimes \tilde{\zeta}\left(t_{2}-t_{1}\right)-\tilde{\zeta}\left(t_{2}\right) \otimes \tilde{\zeta}\left(t_{1}-t_{2}\right)$. 
One can show that $\tilde{\zeta}(2 n)=0$. Using this we get from (??)

$$
\delta \tilde{\zeta}\left(t_{1}, t_{2}\right)=-\sum_{m, n>0} \tilde{\zeta}(m) \wedge \tilde{\zeta}(n) \cdot\left(I+U+U^{2}\right)\left(t_{1}^{m-1} t_{2}^{n-1}\right) .
$$

where $U$ is the linear operator $\left(t_{1}, t_{2}\right) \rightarrow\left(t_{1}-t_{2}, t_{1}\right)$.

Let $\mathcal{C}_{w}^{(2)}$ be the $\mathbb{Q}$-vector space spaned by $\tilde{\zeta}\left(n_{1}, n_{2}\right), \quad n_{1}+n_{2}=w$ in the Lie coalgebra of framed Hodge-Tate structures (i.e. modulo the products $\tilde{\zeta}\left(n_{1}\right) *$ $\left.\tilde{\zeta}\left(n_{2}\right)\right)$. Set $\mathcal{C}_{n}^{(1)}:=<\zeta(n)>_{\mathbb{Q}}$ and $\mathcal{C}_{\bullet}^{(1)}:=\oplus \mathcal{C}_{n}^{(1)}$.

\section{Theorem 4.6. (G3)}

a) One has a canonical isomorphism

$$
\operatorname{Coker}\left(\mathcal{C}_{w}^{(2)} \stackrel{\delta}{\longrightarrow}\left(\Lambda^{2} \mathcal{C}_{\bullet}^{(1)}\right)_{w}\right)=H_{\text {cusp }}^{1}\left(S L_{2}(\mathbb{Z}), S^{w-2} V_{2}\right)^{\vee}
$$

b) The kernel of this map is generated by $\tilde{\zeta}(w)$, so it is zero when $w$ is even and one dimensional when $w$ is odd.

Here $V_{2}$ is the standard $S L_{2}$-module of dimension 2. Part a) follows immediately comparing (??) with the Eihler - Shimura theory of periods(see [La]).

From this we immediately conclude that $(k \geq 1)$

$$
\operatorname{dim} \mathcal{C}_{2 k+1}^{(2)}=1, \quad \operatorname{dim} \mathcal{C}_{2 k}^{(2)}=\left[\frac{k-2}{2}\right]-\operatorname{dim} S_{2 k}^{0}\left(S L_{2}(\mathbb{Z})\right)=\left[\frac{k}{3}\right]-1 .
$$

For the $l$-adic version of the story a result similar to (??) was found independentely and simultaniuosly by Y. Ihara and N. Takao (unpublished, see the statement in the appendix to [Ma]). For $2 k=12$ see [Ih2]. (I am grateful to Y. Ihara and M. Matsumoto for this information). Let $d_{w}^{(2)}$ be the quotient of the $\mathbb{Q}$-vector space generated by $\zeta\left(n_{1}, n_{2}\right)$ modulo the products $\zeta\left(n_{1}\right) \zeta\left(n_{2}\right)$ where $n_{1}+n_{2}=w$. L. Euler $([\mathrm{E}])$ discovered that $d_{2 k+1}^{(2)} \leq 1$. Using the double shuffle

relations D. Zagier proved that $d_{2 k}^{(2)}$ is not bigger than the right hand side of (??), and conjectured that this bound is exact. ([Z1-3]).

The Lie algebra $\mathcal{C}(N)_{\bullet}^{\vee} \otimes_{\mathbb{Q}} \mathbb{Q}_{l}$ should be isomorphic to the Lie algebra of the Zariski closure of the image of the canonical homomorphism

$$
\varphi_{N}^{l}: \operatorname{Gal}(\overline{\mathbb{Q}} / \mathbb{Q}) \longrightarrow \operatorname{Der}_{\text {Out }} \operatorname{Lie}_{1}^{(l)}\left(\mathbb{P}^{1} \backslash\left\{0,\left\{\zeta_{N}^{\alpha}\right\}, \infty\right\}\right) .
$$

Here $\left\{\zeta_{N}^{\alpha}\right\}$ is the set of all $N$-th roots of unity.

\section{Acknowledgements}

I would like to thank the Max Planck Institute (Bonn) for hospitality and support. I am grateful to A. A. Beilinson, E. de Shalit, M. Kontsevich, Yu. I. Manin, B. Mazur, D. Zagier and especially G. Frey for encouragement and stimulating discussions. 


\section{References}

[BMS] A. A. Beilinson, R. MacPherson, and V. V. Schechtman, Notes on motivic cohomology, Duke Math. J. 54 (1987), 679-710.

[BGSV] A. A. Beilinson, A. A. Goncharov, V. V. Schechtman, and A. N. Varchenko, Aomoto dilogarithms, mixed Hodge structures and motivic cohomolgy, Grothiendieck Feschtrift, vol. 86, Birkhäuser, 1990, pp. 135-171.

[Bo] A. Borel, Stable real cohomology of arithmetic groups, Ann. Ecole Norm. Sup. (4) 7 (1974), 235-272.

[E] L. Euler, Opera omnia, Ser. 1, Vol XV, Teubner, Berlin 1917, pp. 217-267.

[Br] D. J. Broadhurst, On the enumeration of irreducible $k$-fold sums and their role in knot theory and field theory, preprint, hep-th/9604128.

[G1] A. B. Goncharov, Multiple polylogarithms, cyclotomy and $G L(\mathbb{Z})$, in preparation.

[G2] - Polylogarithms in arithmetic and geometry, Proc. Internat. Congress Mathematicians, vol. 1, 2 (Zrich, 1994), Birkhäuser, Basel, 1995, pp. 374-387.

[G3] _ Multiple $\zeta$-numbers, hyperlogarithms and mixed Tate motives, preprint, MSRI, 058-93, June (1993).

[G4] Multiple polylogarithms at roots of unity and motivic Lie algebras, Proc. of Arbeitstagung 1997, In preprint MPI 97-62 (available at http//www.mpimbonn.mpg.de/).

[D] P. Deligne, Le group fondamental de la droite projective moine trois points, In: Galois groups over $\mathbb{Q}$. Publ. MSRI, no. 16 (1989), 79-298.

[Dr] V. G. Drinfeld, On quasi-triangular quasi-Hopf algebras and some group related to clousely associated with $\operatorname{Gal}(\overline{\mathbb{Q}} / \mathbb{Q})$, (In Russian) Algebra i Analiz 2 (1990), 149-181, translation in: Leningrad Math. J. 2 (1991), 829-860.

[Ih] Y. Ihara, Braids, Galois groups, and some arithmetic functions, Proc. Internat. Congress Mathematicians, Vol. I, II (Kyoto, 1990), 99-120, Math. Soc. Japan, Tokyo, 1991.

[Ih2] - The Galois representations arising from $\pi_{1}\left(P^{1} \backslash\{0,1, \infty\}\right)$ and Tate twists of even degree, In: Galois groups over $\mathbb{Q}$. Publ. MSRI, no. 16 (1989), 299-313.

[Kr] D. Kreimer, Renormalisation and knot theory, preprint Univ. of Mainz, 1996.

[L] S. Lang, Introduction to modular forms, Springer, Berlin-Heidelberg-New York, 1976.

[Man] Yu. I. Manin, Parabolic points and zeta functions of modular curves, Math. USSR Izvestia 6 (1972), 19-64.

[Ma] M. Matsumoto, On the Galois image in the derivation algebra of $\pi_{1}\left(P^{1} \backslash\{0,1, \infty\}\right)$, Cont. Math., vol. 186, 1995.

[Maz] B. Mazur, Courbes elliptiques et symboles modulaires, Séminaire Bourbaki, 24ème année (1971/1972), Exp. No. 414, pp. 277-294. Lecture Notes in Math., vol. 317, Springer, Berlin, 1973.

[Z1] D. Zagier, Values of zeta functions and their applications, First European Congress of Mathematics, Vol. II (Paris, 1992), 497-512, Progr. Math., 120, Birkhäuser, Basel, 1994.

[Z2] _ Periods of modular forms, traces of Hecke operators, and multiple zeta values, Research into automorphic forms and $L$ functions (Japanese) (Kyoto, 1992), Sūrikaisekikenkyūsho Kōkyūroku No. 843 (1993), 162-170.

[Z3] _ Multiple zeta values, in preparation.

Max-Planck-Instute fur Mathematik, Gottfried Claren str 26, 53225, Bonn Germany and Dept. of Mathematics, Brown University, Providence, RI 02912, USA

E-mail address: sasha@mpim-bonn.mpg.de 